International Journal of Modern Physics B

Vol. 33, No. 6 (2019) 1950028 (21 pages)

(C) The Author(s)

DOI: $10.1142 / \mathrm{S} 0217979219500280$

\title{
Adsorption behaviors of ether and aluminum surface: A molecular dynamics study
}

\author{
Pingan Liu*, Ruochen Sun ${ }^{\dagger}$ and Junpeng $\mathrm{Liu}^{\ddagger}$ \\ College of Aerospace and Civil Engineering, \\ Harbin Engineering University, Nangang District, \\ Harbin City, Heilongjiang Province, P. R. China \\ *liupingan631@163.com \\ †sunrc836@hotmail.com \\ ${ }^{\ddagger}$ pureindigo@hrbeu.edu.cn
}

Received 23 May 2018

Accepted 24 November 2018

Published 3 January 2019

\begin{abstract}
As a pre-study for ether-coated aluminum (Al) nanoparticles (ANPs), ReaxFF or reactive force field-based Molecular Dynamic (MD) Simulations are performed to uncover the mechanism of adsorption behaviors between the Aluminum surface and ether molecules. Meanwhile, part of the results has been verified by experiments. In this study, three different models have been employed with varying concentrations of ether molecules. The obtained results indicate that the adsorption of the ether molecule could be divided into four stages and each stage is associated with charge transfer between Hydrogen and Aluminum atoms. After that, adsorbed ether molecules keep a horizontal state above the Aluminum surface with a vacuum. By evaluating variable temperature conditions, it is concluded that the room-temperature is suitable for forming the ether coating on Aluminum surface. Besides, a higher ether concentration could also bring beneficial effects relating to adsorbing rates. While the disassociated ether solution is removed, it seems that some adsorbed ether molecules will be desorbed, which is similar to the volatilization effect in the filtering experiment. Finally, simulations for desorption show that 455 $(\mathrm{K})$ is a critical point for the adsorbed ether layer.
\end{abstract}

Keywords: Adsorption; coating; molecular dynamic simulations; ReaxFF force field.

PACS numbers: 02.70.Ns, 31.15.Ew, 67.70. $+\mathrm{n}$

\section{Introduction}

In the last two decades, aluminum (Al) nanoparticles (ANPs) have been widely applied as a kind of energetic material in many industries such as pyrotechnics,

This is an Open Access article published by World Scientific Publishing Company. It is distributed under the terms of the Creative Commons Attribution 4.0 (CC-BY) License. Further distribution of this work is permitted, provided the original work is properly cited.

${ }^{\dagger}$ Corresponding author. 
explosives or even the propellant for rockets. $\underline{1}^{-3}$ Ingredients of a modern solid propellant mainly contain seven parts: plasticizer, stabilizer, oxidizer, fuel, binder, curing agent and cross-linking agent,,$\frac{4}{}$ while the ANPs are typical kind of metal fuel among them. The addition of ANPs brings a high energy output, high burning rate, low ignition temperature and ignition time. $\frac{1,5}{2}$ Recently, the advance of forming a coating layer for passivation on the surface of ANPs have attracted researchers' attention. The surface to volume ratio of ANPs is quite high, which implies that the ANPs are more likely to be oxidized and corroded in the real environment. 6 But the ANPs with low active Al continent are toxic for industrial performance. Issues like affecting the aging and ignition phenomenon are both associated with the oxidation of ANPs. ${ }^{7}$ For overcoming such drawbacks, one solution is to coat ANPs by a protection layer. ${ }^{6}$ In this study, an $\mathrm{Al}$ slab is investigated as a simplified model to study adsorption processes.

Various coating materials for protection have been used in previous investigations, such as $\mathrm{Fe}_{3} \mathrm{O}_{4} ; \frac{8}{}$ carbon,, 9 palladium, ${ }^{10,11} \mathrm{Fe},{ }^{12}$ copper, ${ }^{13,14}$ nickel, ${ }^{15,16}$ 1,2epoxyhexane, $\frac{17}{,}$ polytetrafluoroethylene; $\frac{18}{} \mathrm{PMMA}^{19}$ or even $\mathrm{Al}_{2} \mathrm{O}_{3}$ itself. $\stackrel{20-22}{-}$ In this study, ether (chemical constitution formula: $\mathrm{CH}_{3} \mathrm{CH}_{2} \mathrm{OCH}_{2} \mathrm{CH}_{3}$ ) solution is selected as the coating material for Al surface. Same with ANPs, ether has been applied in many industries as well, such as the colorant, spicery, and colorant products. The most critical benefit of ether is that it can also be used as an alternative fuel. ${ }^{23}$ If ether could be successfully coated on ANPs, the obtained particles would have much potential to be the next-generation propellant for the aerospace industry, as this type of newly coated nanoparticles ensures both corrosion resistance and energy transfer simultaneously.

The kinetics of adsorption could be simply divided into four stages, including external diffusion, internal diffusion, surface diffusion and adsorption elementary process. ${ }^{24}$ Molecular dynamic (MD) simulations in this study, is a multi-functional tool to investigate those stages of adsorption and diffusion behaviors at the atomic scale. ${ }^{25-27}$ On the one hand, MD simulations can provide three-dimensional graphs in picoseconds which brings high temporal resolution, $\frac{28}{2}$ on the other hand, several computational properties of the studied system can be obtained by MD simulations as well. $\frac{29}{}$ Previously, MD simulations have already been processed to study adsorbing or coating processes with different materials. For example, self-diffusions of different solution systems were studied with absence or presence of metal surfaces, $\frac{30}{}$ while another research focused on the thermal and energetical performance of coated structure. ${ }^{11}$ Besides, effects of rising temperature and the destroying point for explicit core-shire nanopowders could be discussed by MD simulations as well. $\underline{9}$ Thus, studying the adsorption behavior of ether and $\mathrm{Al}$ surface can give more information before preparing the ether-coated ANPs in the experiment. Apart from MD simulations, adsorption behaviors could also be discussed by Grand Canonical Monte Carlo simulations. $\frac{31}{1}$

It is known that ether is a neutral liquid at room-temperature and the $\mathrm{pH}$ value of which is around 7 . So, it is an embryo deduction that ether will not react 
with $\mathrm{Al}$ powders. To the best of our knowledge, however, there is still little relative literature which introduces interactions between ether and $\mathrm{Al}$ products. Meanwhile, MD simulations studies about coating ANPs by organic solutions are missing as well. Thus, this investigation aims to discuss the mechanism of ether adsorbed by $\mathrm{Al}$ surface and how temperature or density of the solution affects the adsorption behavior. Next, changes in adsorbed configurations are also investigated when the solution is removed or the whole coating system is heated. After all, the coated ANPs are produced and characterized in the lab to further verify the existence of adsorption behavior between ether and $\mathrm{Al}$ surface.

\section{Methods and Setup}

\subsection{MD simulations and ReaxFF force field}

In terms of the setting of MD simulations, there are several alternative force fields, and the one applied in this study is the ReaxFF or reactive force field. The basis of ReaxFF is the concept of bond order, which is introduced by Tersoff ${ }^{32}$ and provides a judgment for the form or break of bonds. ${ }^{28}$ Other than conventional potentials, interatomic bonds are unnecessary to be predefined in ReaxFF package. Instead, interatomic distances are used to evaluate reactions between atoms. While in the multi-element simulations, ReaxFF-based MD simulations are much more effective than Quantum Mechanics computations, as it is an empirical potential. The specified ReaxFF in this study was coded by Hong, ${ }^{33}$ which is developed for a wide variety of organic and inorganic molecular structures. ${ }^{34}$ The development of this force field depends on quantum mechanics-based training to describing the bond breaking and formation of following elements: aluminum $(\mathrm{Al})$; carbon $(\mathrm{C})$; hydrogen $(\mathrm{H})$; and oxygen $(\mathrm{O})$. The calculation of total energy through ReaxFF could be described as follows:

$$
\begin{aligned}
E_{\mathrm{system}}= & E_{\mathrm{bond}}+E_{\mathrm{over}}+E_{\mathrm{under}}+E_{\mathrm{lp}}+E_{\mathrm{val}}+E_{\mathrm{tors}}+E_{\mathrm{vdWaals}} \\
& +E_{\text {Coulomb }}
\end{aligned}
$$

where $E_{\text {system }}$ is the total energy, $E_{\text {bond }}$ is the bond energy, $E_{\text {over }}$ is the overcoordination energy, $E_{\text {under }}$ is the undercoordination energy, $E_{\mathrm{lp}}$ is the lone-pair energy, $E_{\mathrm{val}}$ is the valence angle energy, $E_{\text {tors }}$ is the torsion angle energy, $E_{\mathrm{vdWaals}}$ is the van der Waals energy; $E_{\text {coulomb }}$ is the coulomb energy. As it shows, ReaxFF potential also considers non-bonded effects, like van der Waals and Coulomb interactions. The platform for processing MD simulations and ReaxFF is LAMMPS, $\frac{35}{2}$ which is an open source program mainly developed for MD simulations application. Besides, three initial configurations as shown in Fig. 1 are read as datafiles for LAMMPS, and the creation of datafile is achieved by the "TopoTools" in VMD ${ }^{36}$ software. All configurations of the simulated systems are presented by another visualizing software: OVITO. .37

The Al cube $(3.1 \times 3.1 \times 3.1 \mathrm{~nm})$ is created initially, and above which, a single ether molecule [Fig. 1(a)] or group of 100/150 ether molecules [Figs. 1(b)/1(c)] are 

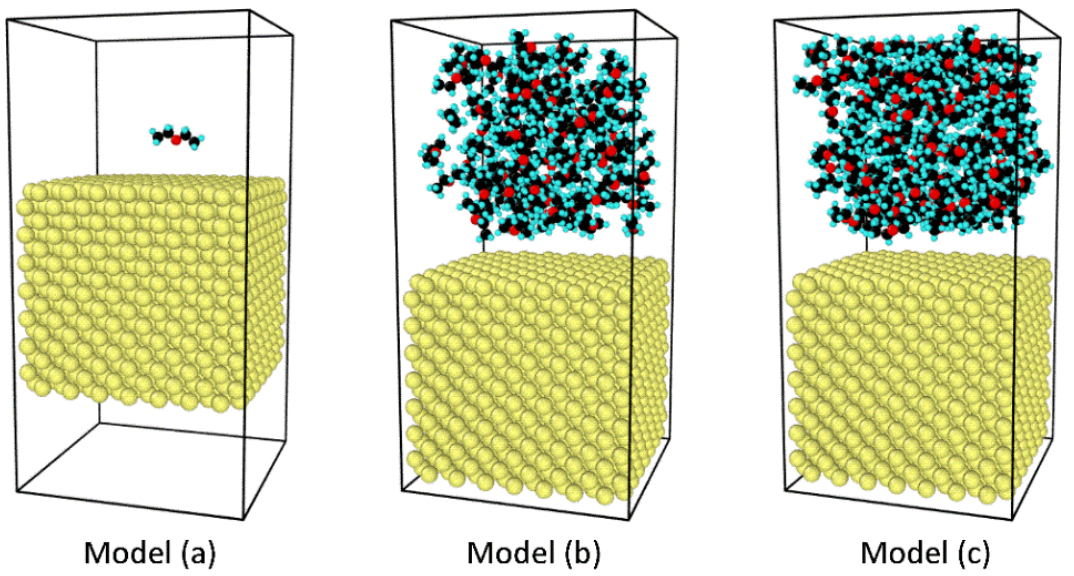

Fig. 1. (Color online) System configurations: Al cube and (a) 1, (b) 100 and (c) 150 ether molecules.

Table 1. Color information for each type of atom.

\begin{tabular}{lll}
\hline Atom type & \multicolumn{1}{c}{ Color } & Color code \\
\hline Aluminum & Yellow & $\#$ d8d86b \\
Carbon & Black & $\# 000000$ \\
Hydrogen & Aquamarine & $\# 33 f f f f$ \\
Oxygen & Red & $\#$ ff0000 \\
\hline
\end{tabular}

set inside the simulating box with periodical boundaries. Parameters of periodical boundaries are about $3.2 \times 3.2 \times 6.4 \mathrm{~nm}$, and colors for each atom type are introduced in Table 1. Ether molecules are placed randomly inside the simulation system. The density of ether solution for Model (b) and (c) are about 0.474 and $0.711 \mathrm{~g} / \mathrm{cm}^{3}$, respectively. The latter density is close to the value obtained by real experiment. Between the $\mathrm{Al}$ cube and ether solution, there is a $3.1 \times 3.1 \times 0.6 \mathrm{~nm}$ vacuum space. In other words, the initial gap between $\mathrm{Al}$ surface and the ether solution is about $0.6 \mathrm{~nm}$. The crystal style for Al cube is fcc and the lattice constant of which is set as 4.05. In LAMMPS, there are at least three methods to control the temperature

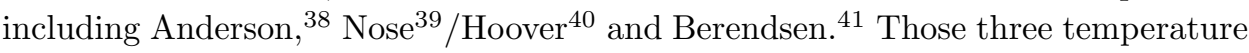
control methods were tested in previous simulations. The controlling theory in this simulation is Nose/Hoover and the temperature damping parameter is $10 \mathrm{fs}$. During the adsorption process, adsorption temperatures are varied for each model. Their specific values are shown in Table 2. For describing the adsorption more effectively, the computed timestep is about $0.1 \mathrm{fs}$ and results are dumped every 100 steps. The total simulations' time length for model (a) is about $6 \mathrm{ps}$, while for the model (b) and (c) it is about 100 ps.

Note that, four parallel simulations are set for the model (b) with four temperature gradients, aiming to investigate the effect of temperature on adsorption 
Table 2. Adsorption temperature for each model.

\begin{tabular}{ccc}
\hline Type of model & Number of ether molecules & Adsorption temperature (K) \\
\hline (a) & 1 & 300 \\
(b) & 100 & $200 / 300 / 500 / 700$ \\
(c) & 150 & 300 \\
\hline
\end{tabular}

behaviors. The reason why the lowest temperature was not $100 \mathrm{~K}$ is that ether solution would be solidified at that experimental condition. Finally, as the adsorption behavior in the study is mainly described as a function of temperature, the canonical ensemble (NVT) is applied to all simulations and the 30 ps equilibration.

\subsection{Density functional theory}

Density functional theory (DFT) calculations are widely performed to test the validity of ReaxFF force field. In this study, DFT calculations are performed depending on CASTEP program from Castep Developers Group (CDG). The calculation selects ultrasoft pseudopotentials and is represented by reciprocal space. The approximation level applies generalized gradient approximation-Perdew Burke Ernzerhof (GGA-PBE). A plane wave cut-off energy valued $435 \mathrm{eV}$ approximates the primary setting, while the energy cut-off for the density is 10 times larger. $\stackrel{42}{ }$ Furthermore, three-dimensional periodical boundaries are built for the calculated model in DFT. Generally, the application of DFT in this study is mainly for obtaining an optimized geometry with charge values for each atom. The structure configuration and charge information of single ether molecule are shown in Fig. 2 and Table 3.

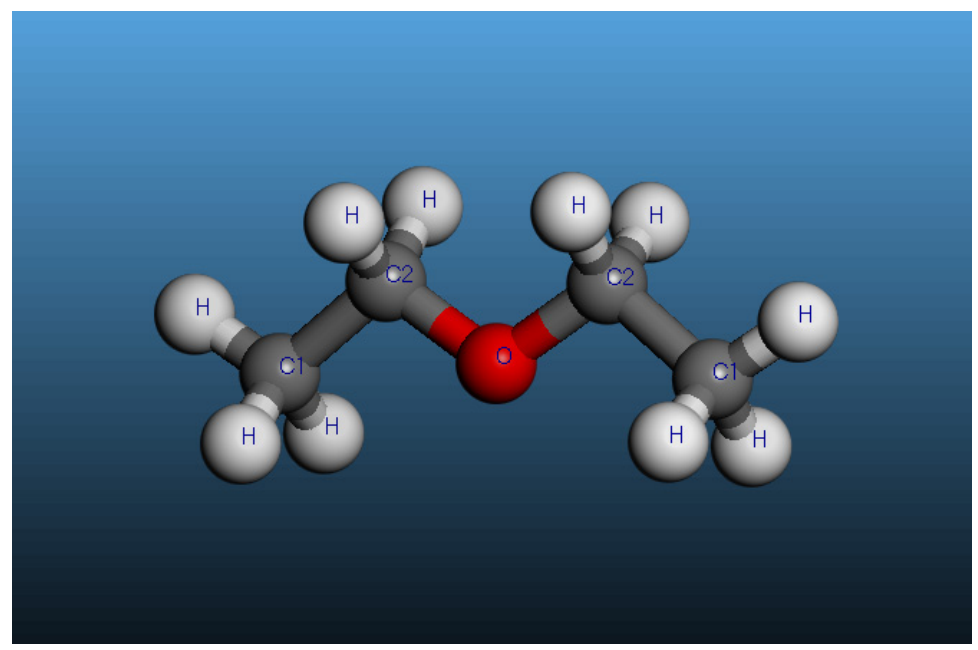

Fig. 2. (Color online) Structure configuration of ether molecule. 
Table 3. Charge information of ether molecule.

\begin{tabular}{cc}
\hline Type of elements & Proton charge $(\mathrm{q} / \mathrm{e})$ \\
\hline $\mathrm{O}$ & -0.32 \\
$\mathrm{C} 1$ & -0.519 \\
$\mathrm{C} 2$ & 0.054 \\
$\mathrm{H}$ & 0.053 \\
\hline
\end{tabular}

\subsection{Characterization of adsorption structure}

For testing and verifying the adsorption between ether and Al surface, the ANPs are coated by ether solution as passivation layers in the experimental condition. Cross-section morphologies of self-produced ether-coated ANPs are investigated by transmission electron microscopy (TEM, Hitachi Limited H-7650), by an accelerating voltage of about $300 \mathrm{kV}$, the aim of which is observing the formation of core-shire structure, so that happening of adsorption could be verified. As the observation should be focused on a single nanoparticle, some acetone solution is added to scatter the nanoparticle. Chemical structures of coated ANPs are tested by Fourier Transform Infrared Spectroscopy (FTIR, Model: Nicolet Avatar 370). Powders of ether-coated ANPs and Potassium Bromide (KBr) are mixed according to the ratio $(1: 100), \underline{44}$ then the mixture is pressed into a tablet to be used as the specimen for FTIR.

\section{Results and Discussions}

\subsection{Mechanism of adsorption between single ether molecule and Al surface}

To investigate the mechanism of adsorption process and be focused on the trajectory of an appointed ether molecule, MD simulation basing on ReaxFF force field is processed to describe the model (a) with single ether molecule and the temperature is set as $300 \mathrm{~K}$. Figure 3 shows the trajectory of ether molecule from initial state to 3500 fs.

The adsorption process for ether molecule and $\mathrm{Al}$ surface could be simply divided into four stages. In the first stage, the adsorption is motivated by the electron transfer from edge Hydrogen atoms to $\mathrm{Al}$ atoms on the top surface, during which, the ether molecule rotates from a horizontal state to almost vertical state. Then the ether molecule keeps this vertical state and gets closer to the surface. When the charge of Hydrogen atom at one edge and $\mathrm{Al}$ atom are in equilibrium, the adsorption will occur in the third stage, returning to the horizontal state, and this stage is followed by the electron transfer between other Hydrogen and Al atoms as well. Finally, the ether molecule is eventually adsorbed, and the adsorption distance keeps fluctuating around a certain value. Limited by the resolution of color coding in Fig. 3, the electron transfer in stages 1-4 need to be analyzed quantitively. Figure 4 counts the sum charge value of $10 \mathrm{H}$ atoms and $1687 \mathrm{Al}$ atoms, respectively, 

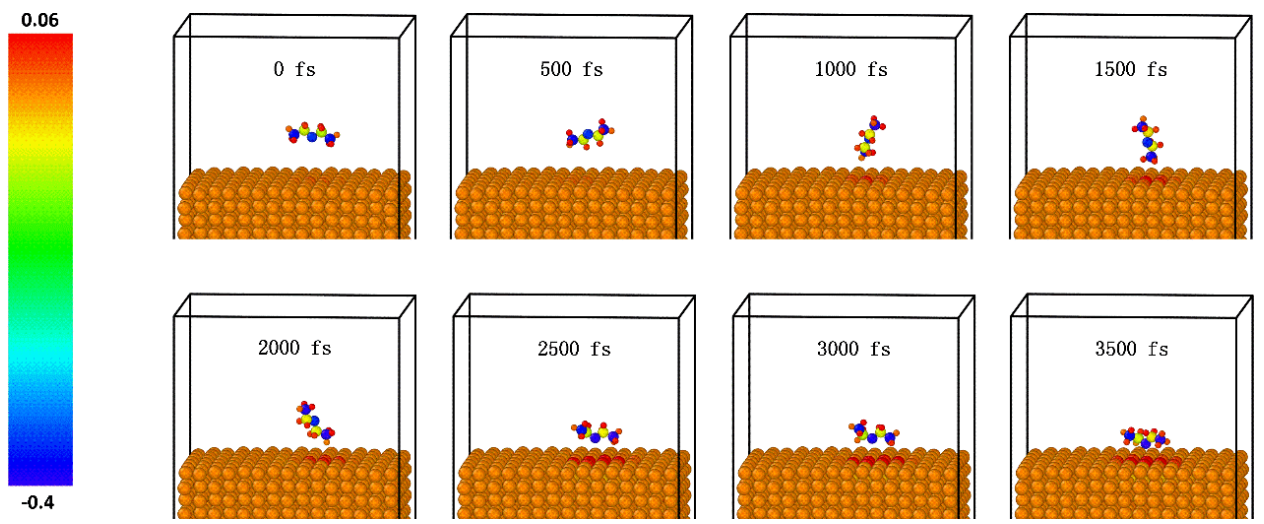

Fig. 3. (Color online) Snapshots of the color gradient (multiple of electron charge) and adsorption process $(0-3500 \mathrm{fs})$ colored by charge value.

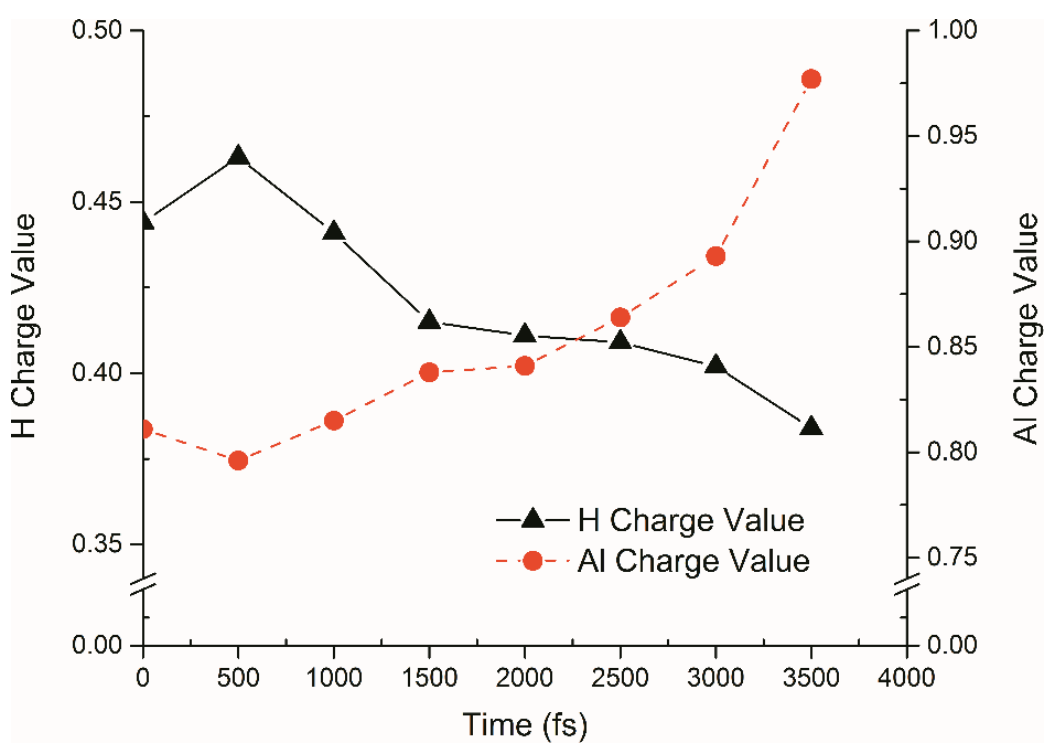

Fig. 4. (Color online) The charge value for hydrogen and aluminum atoms.

to illustrate the transfer of charge in different stages. It shows a correlative relationship between those two types of atoms among four stages. For example, the increase of charge for $\mathrm{H}$ atoms in stage one is accompanied with the decrease of $\mathrm{Al}$ charge value, which generally illustrates that electrons are transferred from Hydrogen atoms to Aluminum atoms in this stage. Those four stages of adsorption are also represented in Fig. 5, which shows the vertical gap distance between ether molecule and $\mathrm{Al}$ surface.

In Fig. 5, the vertical gap distance is determined by the difference between the coordinate of ether molecule and $\mathrm{Al}$ surface. The location of ether molecule 


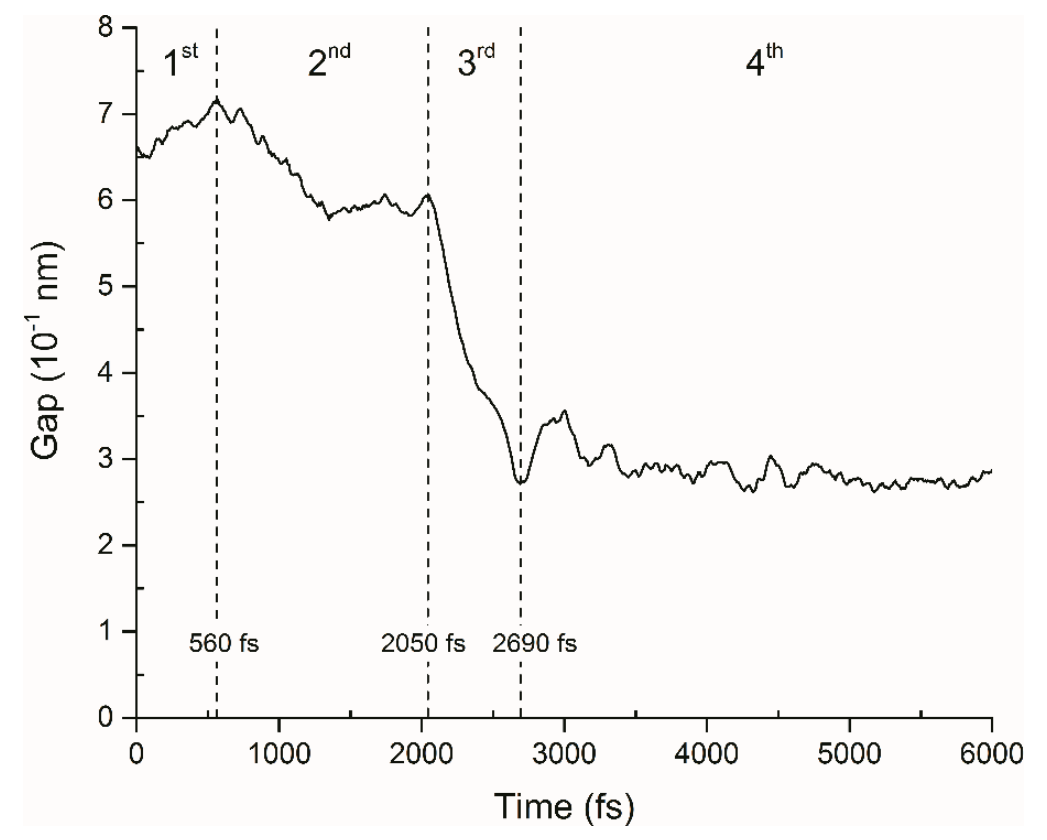

Fig. 5. Change of vertical distance between ether molecule and Al surface.

is represented by Oxygen atom because it is at the geometric center of the whole molecule. Besides, each ether molecule has only one Oxygen atom, and their statistic could be largely simplified. In stage $1(0-560 \mathrm{fs})$, the rise of Z-order position is due to the rotation of ether molecule. It is obviously shown that the adsorbing velocity of stage 3 is higher than stage 2 because the process of rotating back to horizontal happens rapidly. When the system reaches an equilibrium state (after $2690 \mathrm{fs}$ ), the final gap distance of $2.873 \AA$ could be obtained by averaging the value in stage 4 . Since the simulation is in the condition of canonical ensemble, the temperature of the system is fixed during the adsorption, while the temperature setting is firmly related to the kinetic energy. For each atom, the higher the temperature is, the larger the kinetic energy will be. Finally, the study for single ether molecule has been repeated by four parallel MD simulations to confirm its representation.

\subsection{Temperature dependence on adsorption behavior}

According to experimental conditions, Fig. 1(b) is applied for this study, so that the phenomenon from ether solution could be observed. Different from Fig. 1(a), the number of ether molecules has already been changed, so the equilibrium point ought to be reconsidered. By recording the adsorption curve, it is shown in Fig. 6 that the equilibrium point is valid even though there are the same total number of ether molecules in four adsorption systems. Generally, the T2 system consumed the longest time for about 80,000 fs and totally adsorbed 23 ether molecules. Not only 

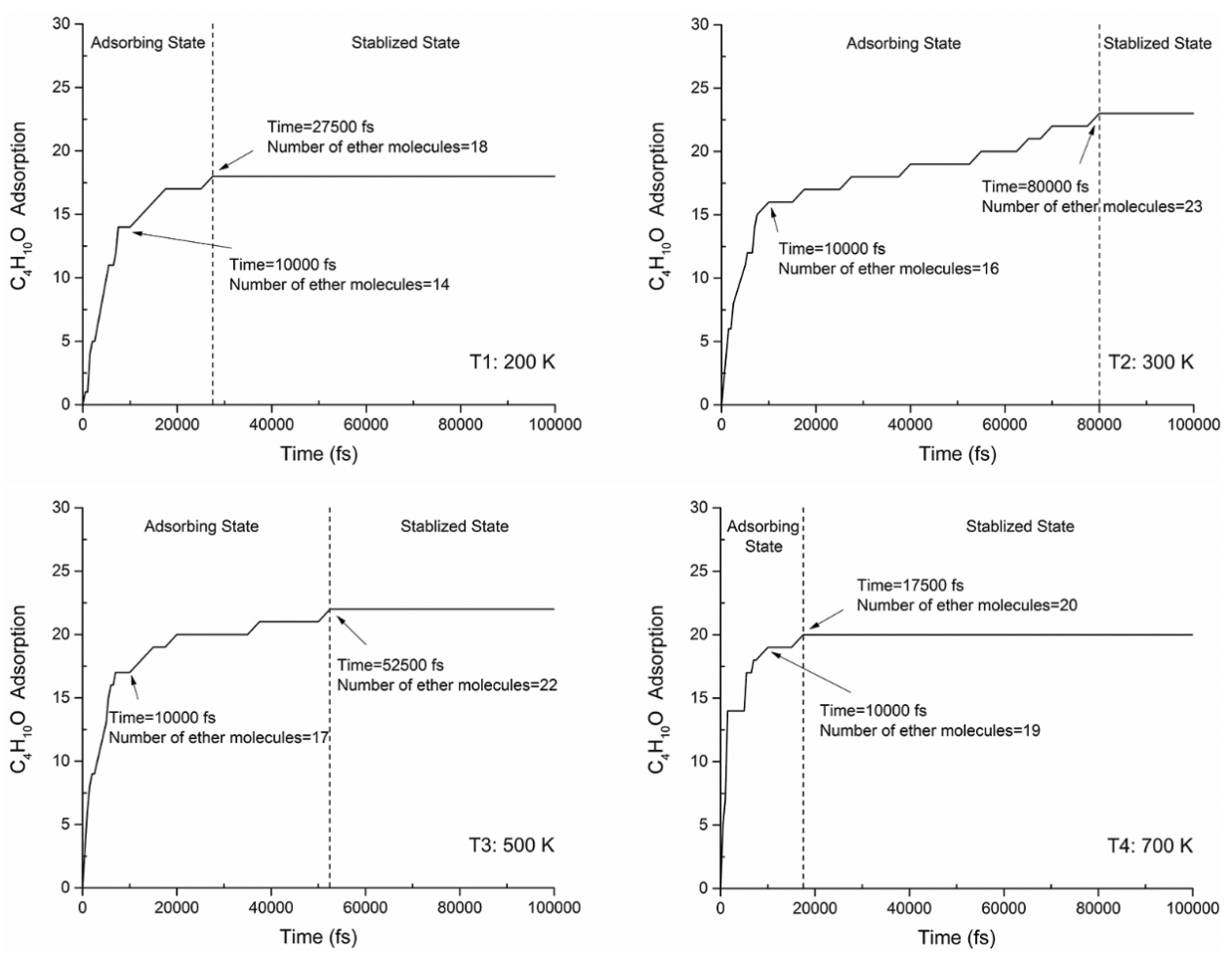

Fig. 6. Number of adsorbed ether molecules on $\mathrm{Al}$ surface varies with time at different temperatures.

for equilibrium but also the number of adsorbed ether molecules, the T2 system kept the highest value among those four parallel simulations. From T2 to T4, the stabilized state is achieved earlier and earlier, and the number of adsorbed ether molecules decreases as well. According to effects of the temperature and kinetic energy, ether molecules would reach the adsorbed position more rapidly at a higher temperature. However, ether molecules with high kinetic energy may constantly restrain the adsorption behavior as it would be harder for the molecule to be captured. Compared with the other three, $\mathrm{T} 1$ system adsorbs the least number of ether molecules within the shortest time, this is because the temperature setting of the T1 system is close to the experimental solidifying point $(157 \mathrm{~K})$ of the ether solution. The kinetic energy of ether at T1 temperature is quite low, although it is still in the liquid state. Nevertheless, in each early stage of the adsorption (before 10,000 fs, Fig. 6), the adsorption curve increases almost linearly at certain temperature.

According to Fig. 6, it is proved that those four adsorption systems have already approached the stabilized state after $80,000 \mathrm{fs}$, so morphologies of four parallel controlled simulations at this moment are selected to present the result of adsorption based on the condition of ether solution. It could be explicitly observed in Fig. 7 that, each of those four snapshots has an adsorbed layer $(3.1 \times 3.1 \times 0.2 \mathrm{~nm})$ above 


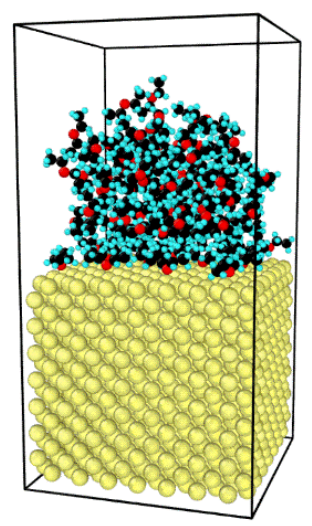

(1) $\mathrm{T}=200 \mathrm{~K}$

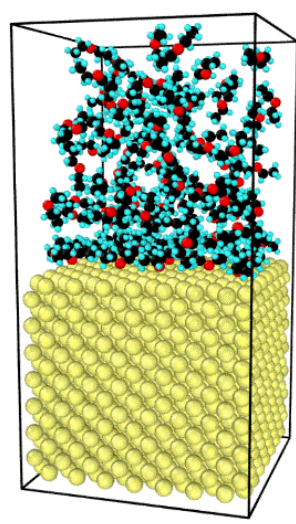

(3) $\mathrm{T}=500 \mathrm{~K}$

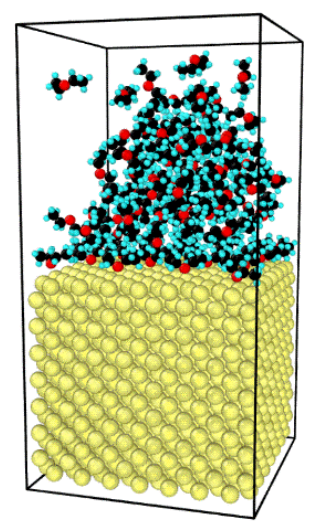

(2) $\mathrm{T}=300 \mathrm{~K}$

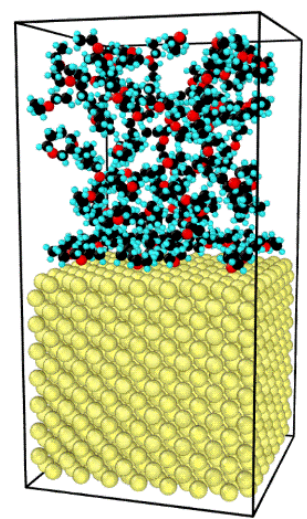

(4) $\mathrm{T}=700 \mathrm{~K}$

Fig. 7. (Color online) Final three-dimensional snapshots $(t=80,000 \mathrm{fs}$ ) of adsorption system at different temperatures.

the Al surface. Same with model (a), there is a vacuum between the Al surface and adsorbed layer as well. In this study, the model is set as a cube with a constant area of the basal surface, so the scale of the vacuum could be simply represented by the height and this result could be a reference for sphere model in the future work. Currently, a larger value of height means a larger scale for vacuum. The height of those vacuums, which is obtained after the adsorption process, are evaluated in Table 4. The scale of the vacuum between adsorption layer and Al surface is not determined by the number of ether molecules or the adsorbing temperature, as the extent of deviations among them are less than $0.297 \AA$.

Configurations of adsorbed layers are single-layer adsorptions, in other words, there is only one layer of ether molecules covering the $\mathrm{Al}$ surface. But it is not enough to demonstrate the existence of adsorbed layer only by OVITO configurations. To show the difference between adsorption layer and disassociated solution of the whole 
Table 4. The height of each vacuum based on different adsorbing models or temperatures.

\begin{tabular}{cccc}
\hline Model & Temperature $(\mathrm{K})$ & Number of ether molecules & Height of vacuums $(\AA)$ \\
\hline (a) & 300 & 1 & 2.873 \\
(b) & 200 & 100 & 2.604 \\
(b) & 300 & 100 & 2.745 \\
(b) & 500 & 100 & 2.576 \\
(b) & 700 & 100 & 2.812 \\
\hline
\end{tabular}

system, mean square displacement (MSD) in the first 5,000 fs is computed in those simulations. Further, their diffusion coefficients (DCs) are computed basing on MSD data. Despite part of sub-diffusions, diffusion behaviors of particles are generally computed in the simple diffusion mode to describe their Brownian movements in this MD study. Although the number of adsorbed ether molecules had not reached the maximum value at this moment, the difference of MSD plot is also shown explicitly in Fig. 8. Through Eq. (2) below, numerical variances of DC for adsorbed layer or free ether molecules are shown in Table 5 . From this table, the DC for disassociated molecules is about $0.56 \times 10^{-4} \mathrm{~cm}^{2} / \mathrm{s}$ at $200 \mathrm{~K}$ and which is about 2.33 times larger than those for adsorbed molecules. Which means that the diffusing
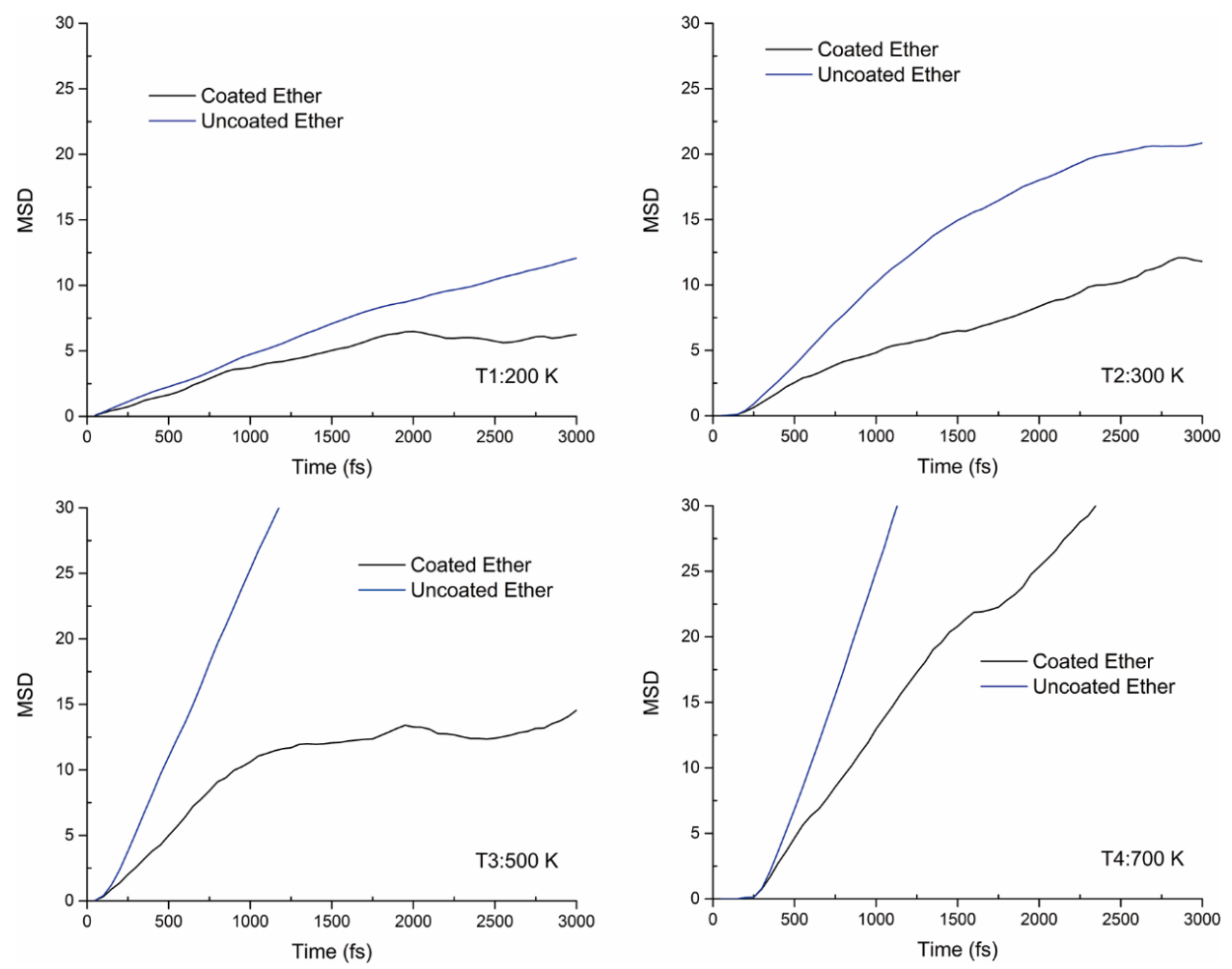

Fig. 8. (Color online) MSD plots for adsorbed and disassociated ether molecules for T1, T2, T3 and T4 systems. 
Table 5. DC values for adsorbed and disassociated ether molecules in adsorption systems for $\mathrm{T} 1, \mathrm{~T} 2, \mathrm{~T} 3$ and $\mathrm{T} 4$.

\begin{tabular}{ccc}
\hline Adsorption system & $\begin{array}{c}\text { DC for adsorbed ether } \\
\left(1 \times 10^{-4} \mathrm{~cm}^{2} / \mathrm{s}\right)\end{array}$ & $\begin{array}{c}\text { DC for disassociated ether } \\
\left(1 \times 10^{-4} \mathrm{~cm}^{2} / \mathrm{s}\right)\end{array}$ \\
\hline T1 & 0.24 & 0.56 \\
T2 & 0.53 & 1.66 \\
T3 & 0.77 & 2.97 \\
T4 & 1.92 & 5.59 \\
\hline
\end{tabular}

movement of molecules will be weakened after being adsorbed above Al surface, and in this occasion the trajectory of the molecule will be within a certain layer. Comparing T1 to T4, DC of both adsorbed and disassociated molecules get higher, respectively, which demonstrates that the higher the temperature is, the larger the DC of both coated and uncoated ether molecules will be. Furthermore, it seems that the presence of $\mathrm{Al}$ surface could weaken the effect from rising temperature as well, because the extent of changes on disassociated molecules is much higher than those on adsorbed molecules while at a higher temperature. By combining this result with Fig. 6, the effect of high diffusion on adsorption process could be concluded then. In the early stage, the high DC leads more molecules to be captured within the adsorbed layer, however, the negative effect is that some adsorbed molecules are more prone to be disassociated finally. So, keeping the diffusion coefficient around T2 value can be a suitable choice in future study.

$$
\mathrm{MSD}=6 \times D C \times \Delta t .
$$

\subsection{Density dependence on adsorption behavior}

As previous literatures concluded, the adsorbing reaction between oxygen gas and Al surface was greatly affected by the density of oxygen gas, and this result was valid not only in the experiment but also in MD simulations study. ${ }^{22,33,34}$ So, it is essential to investigate if there is a dependence of the density of ether solution on the adsorption of $\mathrm{Al}$ surface as well. To demonstrate the consequence of this discussion, Figs. 1(b) and 1(c) are simulated to make a comparison, as there is an initial gap of ether molecules number. Figure 9 shows general configurations of both models (b) and (c) at 80,000 fs, from which, the extent of diffusion could be directly observed. It seems that ether molecules in model (c) are rather more likely to be scattered, and this consequence is similar to the effect of rising environmental temperature (Fig. 7). Meanwhile, MSD plots in Fig. 10 approve the extent of diffusion from another perspective. It is showed that the MSD value for disassociated ether molecules in model (c) is much higher than that in model (b), so coated ether molecules in model (c) are likely to be stabilized.

Another critical issue of the adsorption is the adsorbing rate, which is closely related to the covering effect of the $\mathrm{Al}$ surface. By evaluating the number of adsorbed ether molecules, it seems that their adsorbing rate is almost equal at the early stage 

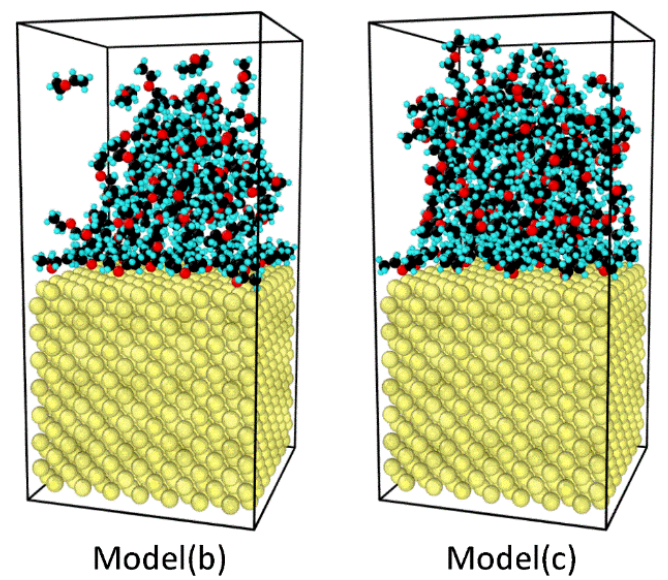

Fig. 9. (Color online) Snapshots $(t=80,000$ fs) of adsorption systems basing on models (b) and (c).

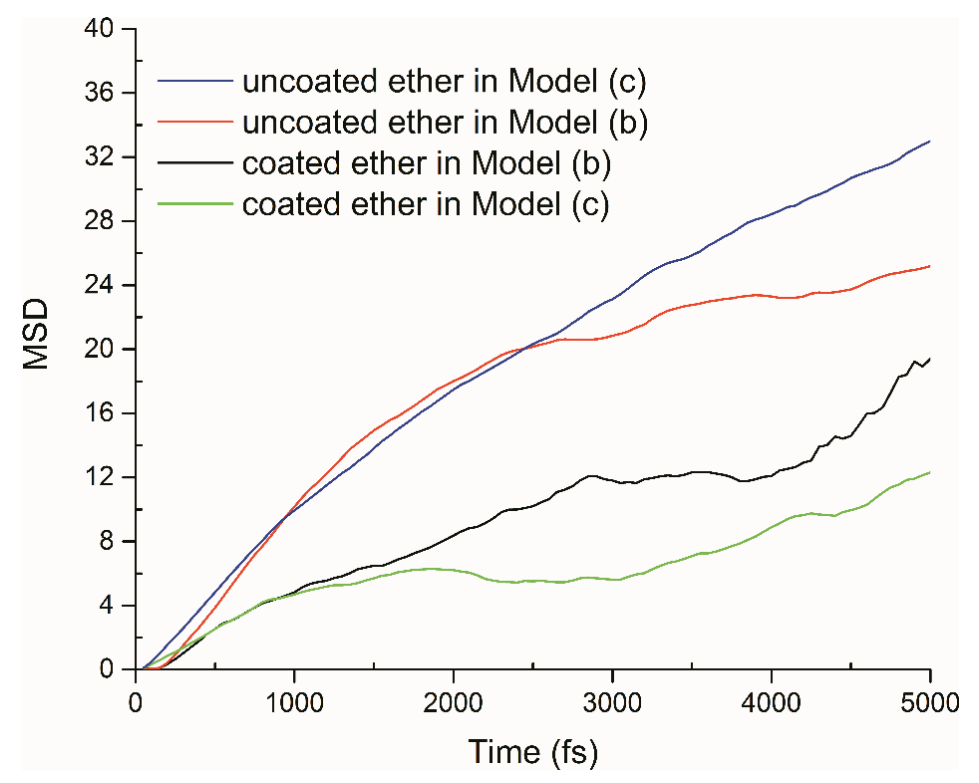

Fig. 10. (Color online) MSD plots for coated and uncoated ether molecules in models (b) and (c) with time.

(before 12,500 fs, see Fig. 11), after that, 50 more initial molecules show their effect on adsorbing rates, although the final gap number of adsorbed ether molecules is implicit. Which means that, an adsorbing system with a higher density of ether might achieve the stabilized state more rapidly, but it will not have much effect on the final adsorbing result. This is because its final quantity is determined by the availability of free adsorbing sites on $\mathrm{Al}$ surface, but not the concentration of ether solution. 


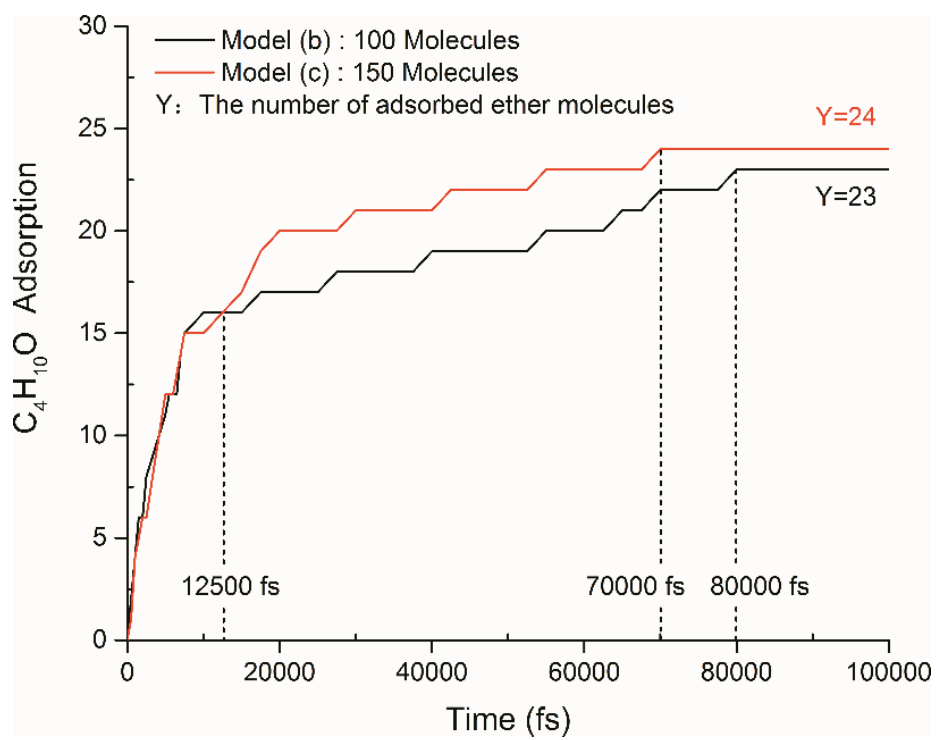

Fig. 11. (Color online) Number of ether molecules adsorbed on Al surface of models (b) and (c) with time.

\subsection{Simulations of filtering and heating processes for ether-coated Al surface}

In the experimental study, when ANPs have been coated in the organic solution, the following operation is always to filter those powders and dry them to obtain a clean core-shire structure. $\frac{5,18,19,45}{5}$ To pave the way of refining the experimental condition, it is critical to simulate the filter process and investigate the change of adsorbed configuration. According to the consequence in Secs. 3.2 and 3.3, model (c) after adsorption is selected to be filtered. This is because the adsorbed layer obtained by model (c) in $300 \mathrm{~K}$ could stably contain a maximum number of ether molecules in this study. The filtering process is simulated by 10 -cycles of removing disassociated ether molecules, and removing operations are processed by "delete atoms" commands in LAMMPS. Those commands are set to be focused on a three-dimensional region beyond adsorbed layer. In this region, ether molecules are not under captured, as they are disassociated ether molecules. In each circle, the deleting command would act on those molecules, and then allow more ether molecules to be disassociated. The time length of each cycle is about 20,000 fs, so that all disassociated ether molecules could leave the adsorbed layer and move into the region for "delete_atoms". The design of this simulation is similar with filtering coated ANPs out of organic solution and then giving them enough time to volatilize part of the organic layers. Finally, the remaining organic layer acts as the passivating coating on ANPs surface. On this occasion, the model system without disassociated ether molecules could be seen as the simulation of dried ANPs after coating. 


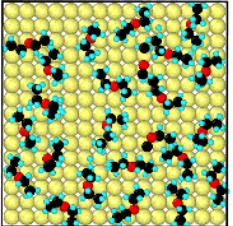

(1)

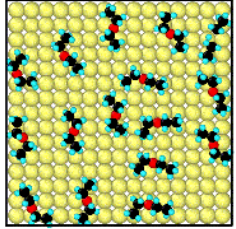

(3)

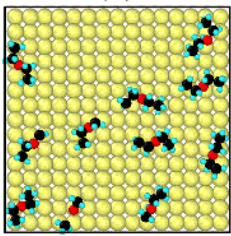

(5)

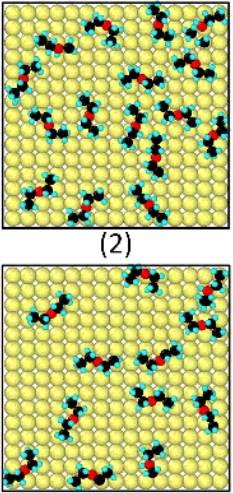

(4)

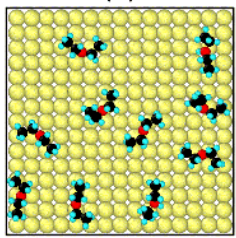

(6)

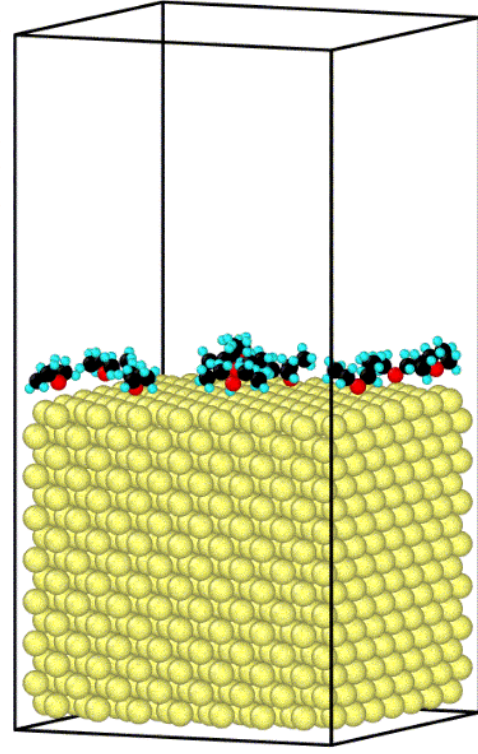

(7)

Fig. 12. (Color online) Top-views of adsorbed layers at different stages: (1) origin; (2) cycle 2; (3) cycle 4; (4) cycle 6; (5) cycle 8; (6) cycle 10 and (7) final three-dimensional snapshot.

In Figs. 12(1)-12(6), modified top views of adsorbed layers are shown at different stages including: origin, cycle 2, 4, 6, 8 and 10, respectively. Those configurations are modified by slicing the region of disassociated molecules, then the cross-section of adsorbed layer could be attained. But limited by the precision of slice, the atomic structure of each molecule may not be shown completely. This is because the main body of this ether molecule is in the adsorbed layer, but one or two of the atoms have been sliced. In this case, the modified ether molecules are still considered to be adsorbed entirely as well. By statistics of the adsorbed ether molecules in the histogram (Fig. 13), the number of which in each stage of cycles could be compared. Although the number of adsorbed ether molecules are cut in more than half, it does not mean that the extent of disassociating ether molecules is equal in the real experiment. But it could be known at least that the filter and dry process will sharply reduce the quantity of adsorbed molecules. Figure 12(7) is an unmodified snapshot, which shows the stable adsorbed ether layer at the end of filtering simulation and there will be no more ether molecules to disassociate at room temperature from then on.

When dried ether-coated ANPs are prepared, a further necessary process before real application is to store those nanopowders well. In other words, the coated configuration should not fail, and the quantity of ether molecules should not decline any more. In this section, the variable quantity to be discussed is the condition of temperature. As Fig. 14 shows, the whole adsorbed structure is heated up to $547 \mathrm{~K}$ 


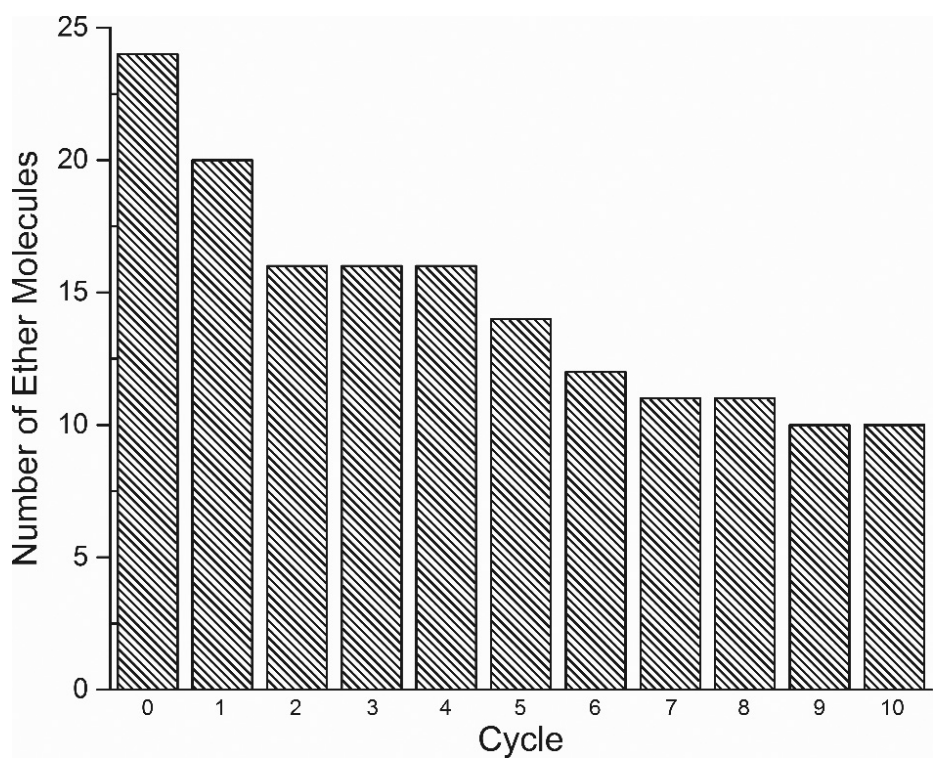

Fig. 13. The number of ether molecules in each stage of cycles.

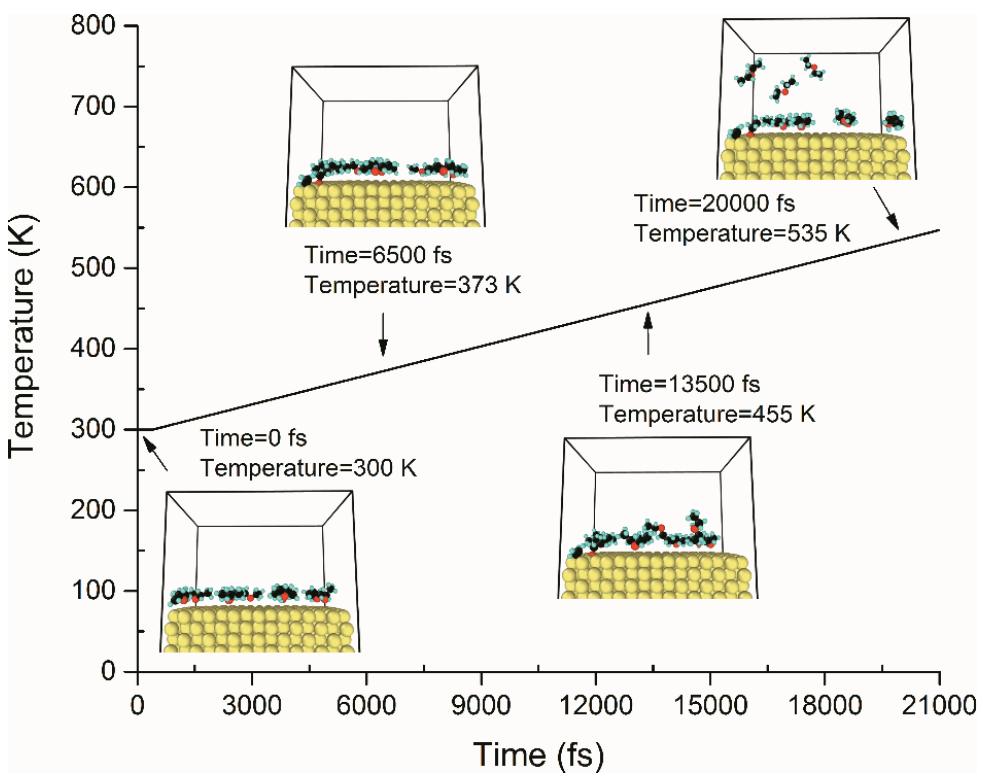

Fig. 14. (Color online) The relationship between adsorbed ether layer and raising temperature.

in 21,000 fs. Despite the first $400 \mathrm{fs}$ for relaxation, the temperature of the structure is raised linearly, and the heating rate is $0.012 \mathrm{~K} / \mathrm{fs}$, which is guided by a previous simulation study. $\frac{46}{}$ By observing the adsorbing state of ether layer, the timestep for the disassociating point could be focused, then the temperature relating to it 


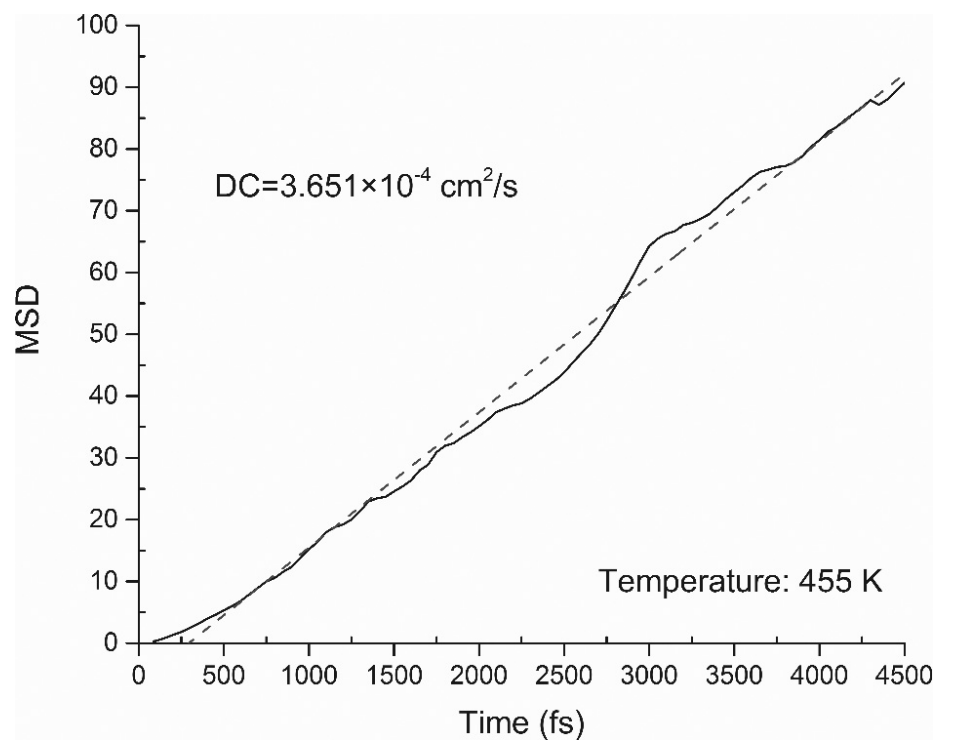

Fig. 15. MSD plot of adsorbed layer without solution at disassociating point with time.

is obtained. It seems that, the adsorbed ether layer would be destroyed while the temperature is heated to $455 \mathrm{~K}$ which means that the condition of temperature for storing ether-coated $\mathrm{Al}$ powder should not be higher than or even close to $455 \mathrm{~K}$. Otherwise, all previous efforts will be wasted. With the help of MSD, diffusion coefficients for disassociating point could be calculated further. It is shown from Fig. 15 that, when the ether solution is filtered, and the diffusion coefficient of adsorbed ether layer reaches $3.651 \times 10^{-4} \mathrm{~cm}^{2} / \mathrm{s}$, the adsorbing energy will be unable to maintain the behavior of adsorption. As a result, those ether molecules will be back to free state again.

\subsection{Characterization of Coated ANPs}

It was reported that the boiling point of ether is about $307.63 \mathrm{~K}, \underset{47}{ }$ which means that the solution of ether is easy to be volatilized even at room-temperature. This is quite unbeneficial for the application of coating material, and the experimental verification turns to be more necessary for this study. Coated ANPs to be tested are prepared in the laboratory of Harbin Engineering University. Figure 16 demonstrates the XRD plot of ether-coated ANPs, in which both $\mathrm{Al}$ phase and $\alpha-\mathrm{Al}_{2} \mathrm{O}_{3}$ phase are identified with peaks at $38.56^{\circ}, 44.6^{\circ}, 65.18^{\circ}, 78.3^{\circ}$ and $82.52^{\circ}$. So, it is confirmed that there is no metal impurity in $\mathrm{Al}$ core, while the organic coating shine is identified by FTIR which could analyze the chemical bonding of processed powders. As Fig. 17 shows, there are five characteristic adsorption peaks in the spectrum of core-shire style powders. Their parameters agree with results obtained by previous study. ${ }^{21,48,49}$ The characteristic of ether bond $(\mathrm{C}-\mathrm{O}-\mathrm{C})$ is represented by 


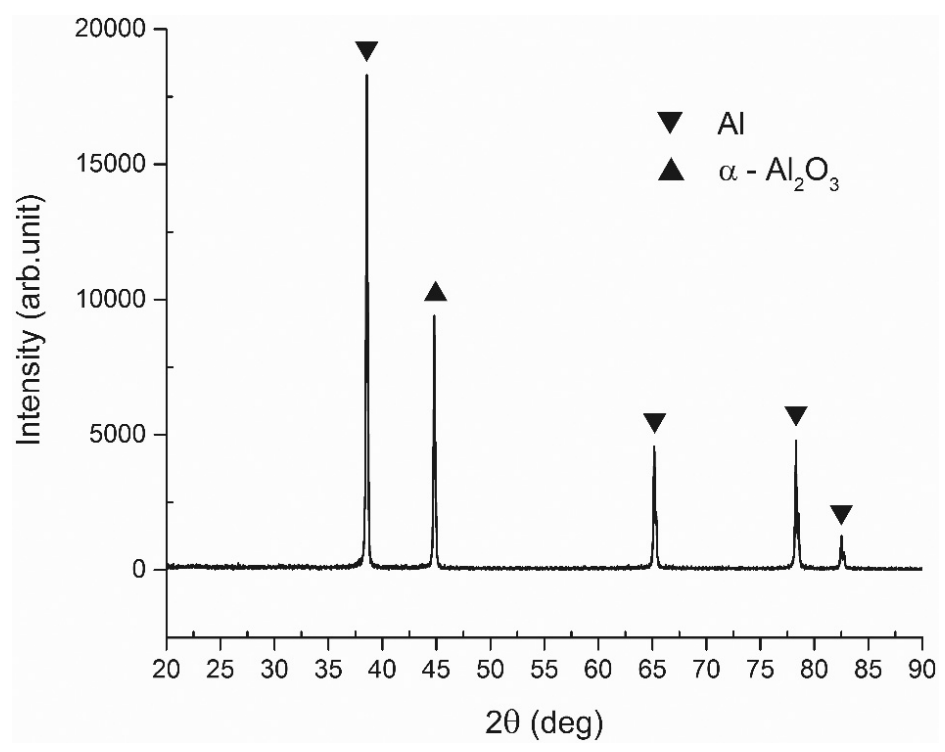

Fig. 16. XRD patterns of ether-coated Al nanopowders.

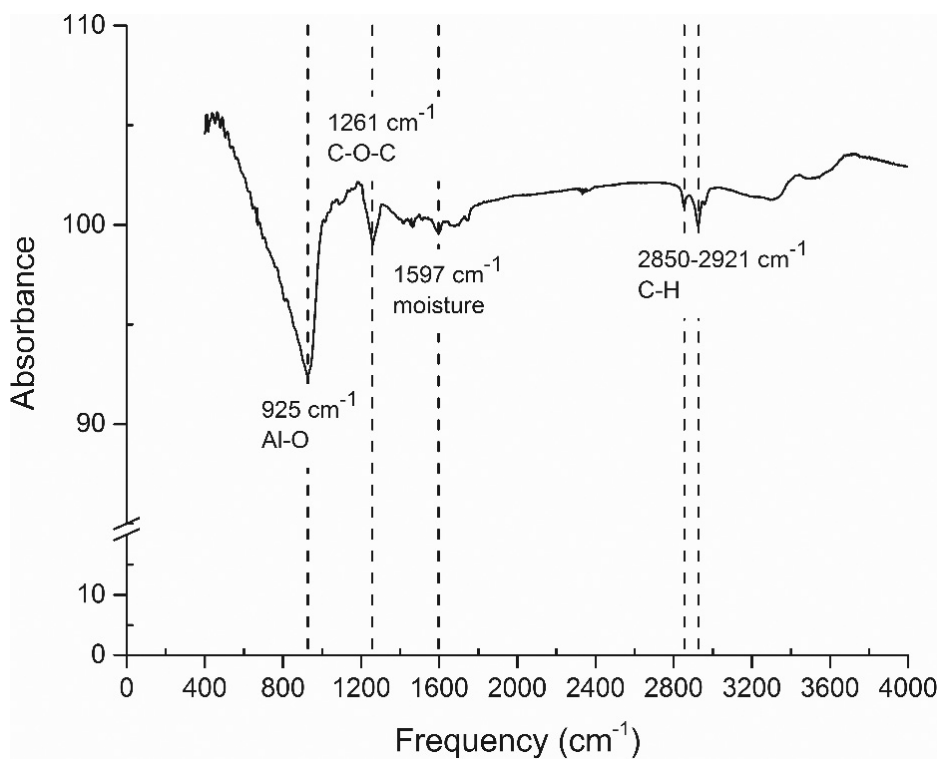

Fig. 17. FTIR analysis of ether-coated Al nanopowders.

the adsorbing peak at $1261 \mathrm{~cm}^{-1}$. $\underline{48}$ Besides, adsorbing peaks in the range between 2850 and $2921 \mathrm{~cm}^{-1}$ are led by the stretching vibrations of $\mathrm{C}-\mathrm{H}$ as well. ${ }^{49}$ According to the ReaxFF-based MD simulations results, it seems that whole structures of ether molecule are not broken up after being adsorbed. Similar with XRD result, 


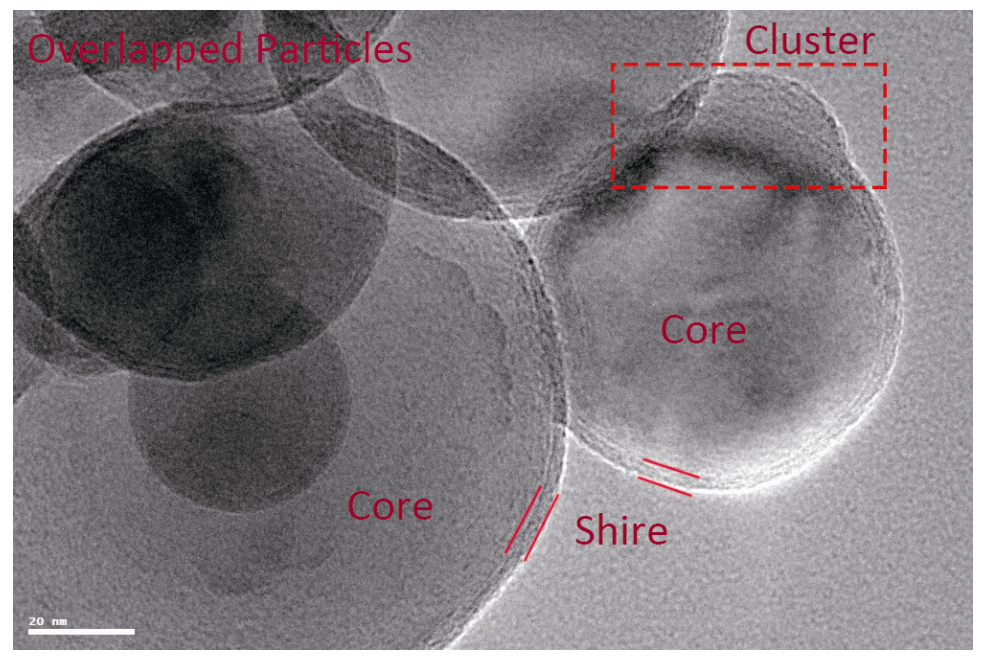

Fig. 18. (Color online) TEM image of ether-coated Al nanopowders.

large quantities of Al-oxide bonds are formed in the coated structure, as there is a large peak at $925 \mathrm{~cm}^{-1}$. The source of oxygen atom should not be from ether molecules, because the molecule did not disintegrate during the adsorbing process. So, it is deduced that, the ANPs were partly oxidized before the coating operation in this experiment. Apart from the oxidation, the presence of moisture is also detected weakly around $1597 \mathrm{~cm}^{-1} \cdot 21$ Although the ether is coated successfully on ANPs to form ether-coated ANPs, the experimental operation still requires to be refined.

General two-dimensional graphs of coated ANPs are demonstrated by TEM in Fig. 18. This nanoconfiguration reveals that there is a layer adsorbed on the surface of ANPs. Besides, the formation of layer is not uniform, as there is a layer of clusters on a single particle. According to the result by XRD, FTIR and TEM, it is reasonable to corroborate that there is a layer of ether adsorbed on $\mathrm{Al}$ nanopowders, just like what was presented in MD simulations. Further, the mechanism of ether cluster on the Al surface could be investigated by MD simulations in the future.

\section{Conclusion}

We performed ReaxFF MD simulations to study adsorption processes of ethercoated Al slab. Also, the experimental characterization is processed for verification. The aim of present work is to lay the groundwork for producing ether or other organic mixture-coated ANPs in the future, and that would be a critical step for the next-generation propellant. The state of adsorption in this study is presented by graphs based on OVITO and TEM, from which the formation of adsorbed layer could be explicitly observed. Through this study, the result and conclusion of the ether- $\mathrm{Al}$ adsorbing system are obtained as follows. Above all, it is noticed that the 
adsorption process is related to charge transfer, especially this kind of transfer is mainly between hydrogen and $\mathrm{Al}$ atoms. This adsorption process could be divided into four stages and each stage is associated with different state of movement, while in the final stage, the ether molecule is more likely to be adsorbed horizontally, and the Oxygen atom is placed towards the Al surface. The gap distance is verified to be about $2.873 \AA$. Next, if the temperature of system is set to be a variable quantity, it seems that $300 \mathrm{~K}$ could be a better setting value compared with higher or lower parameters, because there are more ether molecules adsorbed on the surface in $300 \mathrm{~K}$ than those in other temperature conditions. However, similar results could not be obtained from the higher density of ether solution. The change caused by higher ether concentration is about the adsorbing rate in this study. Next, simulations of filtering and heating operation reveal that the loss of molecular pressure can lead to disassociations of adsorbed ether molecules, and those remaining molecules are still going to be disassociated as well while being heated up to about $455 \mathrm{~K}$. Finally, our experiments show that there is an ether layer successfully coated on ANPs, and this phenomenon generally has an agreement with simulation results.

\section{Acknowledgment}

This investigation is supported by Fundamental Research Funds of Harbin Engineering University (No. HEUCFP201780). The authors are also very grateful to Professor Jingyuan Liu, Zhenyuan Bai (College of Material Science and Chemical Engineering, Harbin Engineering University) and Tao Ding, Ting Wang (College of Aerospace and Civil Engineering, Harbin Engineering University) for their technical aids on TEM, FTIR and XRD, respectively.

\section{References}

1. L. Meda et al., Mater. Sci. Eng. C 27, 1393 (2007).

2. E. L. Dreizin, Prog. Energy Combustion Sci. 35, 141 (2009).

3. A. Gromov et al., Int. J. Energetic Mater. Chem. Propulsion 13, 399 (2014).

4. S. Chaturvedi and P. N. Dave, Arabian J. Chem. (2015), http://dx.doi.org/10.1016/ j.arabjc.2014.12.033.

5. R. J. Jouet et al., Am. Chem. Soc., doi:10.1021/cm048264y.

6. L. Guo et al., Appl. Surf. Sci. 254, 2413 (2008).

7. A. B. Vorozhtsov et al., Thermochimica Acta 636, 48 (2016).

8. L. Yuan et al., J. Alloys Compd. 583, 492 (2014).

9. Q. Yi et al., Chem. Phys. Lett. 669, 192 (2017).

10. J. Z. Wen et al., J. Nanopart. Res. 16, 1 (2014).

11. N. H. Nguyen et al., Chem. Phys. Lett. 503, 112 (2011).

12. Z. Wang et al., Adv. Powder Technol. 25, 676 (2014).

13. Y. Wang et al., Thermochimica Acta 463, 69 (2007).

14. K. Lee et al., Combustion Flame 162, 3823 (2015).

15. D. S. Sundaram, P. Puri and V. Yang, J. Nanopart. Res. 16, 1 (2014).

16. D. S. Sundaram, P. Puri and V. Yang, J. Phys. Chem. C 117, 7858 (2013).

17. P. A. Jelliss et al., Solid State Sci. 23, 8 (2013). 
18. K. T. Kim et al., Mater. Lett. 167, 262 (2016).

19. H. Liu, H. Ye and Y. Zhang, Appl. Surf. Sci. 253, 7219 (2007).

20. D. Wang et al., Appl. Cataly. A, General 527, 60 (2016).

21. S. Ghosh et al., Ceram. Int. 37, 1115 (2011).

22. N. Cai and G. Zhou, Phys. Rev. B 84, 125445 (2011).

23. O. I. Awad et al., Renew. Sustainable Energy Rev. 82, 2586 (2018).

24. A. Dabrowski, Adv. Colloid Interface Sci. 93, 135 (2011).

25. S. Wang, F. Javadpour and Q. Feng, Fuel 181, 741 (2016).

26. S. Wang et al., Energy Fuels, doi:10.1021/acs.energyfuels.7b02892.

27. S. Wang et al., Int. J. Coal Geol. 147-148, 9 (2015).

28. J. Liu and X. Guo, Fuel Process. Technol. 161, 107 (2017).

29. Y.-Y. Li et al., Fuel 193, 331 (2017).

30. K. Kholmurodov et al., Chem. Phys. 402, 41 (2012).

31. S. Wang et al., Chem. Eng. J. 355, 76 (2019).

32. J. Tersoff, Phys. Rev. Lett. 56, 632 (1986).

33. S. Hong and A. C. T. van Duin, J. Phys. Chem. C 119, 17876 (2015).

34. S. Hong and A. C. T. van Duin, J. Phys. Chem. C 120, 9464 (2016).

35. S. Plimpton, J. Comp. Phys. 117, 1 (1995).

36. W. Humphrey, A. Dalke and K. Schulten, J. Molecular Graph. 14, 33 (1996).

37. S. Alexander, Model. Simul. Mater. Sci. Eng. 18, 1 (2010).

38. H. C. Andersen, J. Chem. Phys. 2384, 2384 (1980).

39. S. Nosé, Am. Institute Phys. 81, 511 (1984).

40. W. G. Hoover, Am. Phys. Soc. 31, 1695 (1984).

41. H. J. C. Berendsen et al., J. Chem. Phys. 81, 3684 (1984).

42. I. A. Pašti, N. M. Gavrilov and S. V. Mentus, Adv. Phys. Chem. (2011), doi:10.1155/ $2011 / 305634$.

43. H. J. Monkhorst and J. D. Pack, Phys. Rev. 13, 5188 (1976).

44. X. Wu et al., Comput. Electron. Agri. 147, 64 (2018).

45. H. Cui and G. Hong, J. Mater. Sci. Lett. 21, 81 (2002).

46. N. H. Nguyen and J. Z. Wen, Open Surface Sci. 3, 82 (2011).

47. M. Wojciechowski, Natio. Bureau Standa. 17, 459 (1936).

48. A. Tewatia et al., Mater. Sci. Eng. 216, 41 (2016).

49. K. Chruszcz-Lipska, B. Trzewik and B. Winid, Spectrochimica Acta Part A: Molecular and Biomolecular Spectroscopy 182, 50 (2017). 